\title{
Microsymposia
}

MS.30.3

Acta Cryst. (2011) A67, C78

\section{Diffuse scattering from molecular crystals}

D. J. Goossens, Research School of Chemistry, Australian National University, Canberra(Australia).E-mail: goossens@rsc.anu.edu.au

Diffuse scattering probes the local ordering in a crystal, whereas Bragg peaks are descriptive of the average long-range ordering. Shortrange order can be thought of as local departures from that long-range average. The population of local configurations can be explored by modelling the three-dimensional (3-d) distribution of diffuse scattering. Local configurations are not constrained by the average crystallographic symmetry so one way of modelling diffuse scattering is by modelling a structure containing some disorder (or short-range order) and then calculating its diffuse scattering. The structure must contain enough atoms/molecules to give a statistically valid model of the populations of local configurations.

It is possible to collect three-dimensional (3-d) volumes of diffuse scattering with X-rays or neutrons [1], [2] and reconstruct large volumes of reciprocal space. Increases in computing power allow such data to be modelled in detail, even for complex systems such as molecular crystals, in which the molecules possess both external degrees of freedom (position and attitude of the molecule) and internal (molecular conformation) [3].

Thus paper describes the modelling of the local ordering in molecular crystals including para-terphenyl and ferroelectric triglycine sulphate. This includes a discussion of the different approaches to the analyses and the types of information available, and a brief outline of the software that has been developed to tackle such problems.

[1] E.J. Chan, et al., Acta Crystallogr. 2010, B66 696-707. [2] R.E. Whitfield, et al., Metallurgical and Materials Transactions A, 2011, in press. [3] D.J. Goossens, et al., Metallurgical and Materials Transactions A, 42, 2011 23-31.

Keywords: diffuse scattering, Monte-Carlo, molecular crystals

MS.30.4

Acta Cryst. (2011) A67, C78

A new model of correlated disorder in relaxor ferroelectrics Dmitry Chernyshov, ${ }^{\mathrm{a}}$ Alexi Bosak, ${ }^{\mathrm{b}}$ Sergey Vakhrushev, ${ }^{\mathrm{c}}$ Michael Krisch, ${ }^{b} \quad{ }^{a}$ Swiss-Norwegian Beam Lines, BP 220, 38043 Grenoble Cedex, (France). ' European Synchrotron Radiation Facility, BP 220, 38043 Grenoble Cedex, (France). 'Ioffe Physico-Technical Institute, St.Petersburg, 194021, (Russia). E-mail: dmitry.chernyshov@esrf.fr

Relaxor ferroelectrics are known since more than fifty years and have attracted significant interest because of their numerous unusual properties and a variety of applications ${ }^{1}$; they also show pronounced diffuse scattering directly linked to relaxor physics. One of the most widely used concepts dealing with characteristic diffuse scattering is the model of polar nanoregions (PNR) in relaxors, first proposed in $1983^{2}$.

The model is based on the existence of small regions (down to few unit cells in some dimensions) of local polarization, with the polarization parallel to specific high-symmetry directions. It is commonly accepted that the strong neutron- and x-ray diffuse scattering in the vicinity of Bragg reflections is a signature of $\mathrm{PNRs}^{3}$. We present new diffuse scattering maps for $\mathrm{PbMg}_{1 / 3} \mathrm{Nb}_{2 / 3} \mathrm{O}_{3}-\mathrm{PbTiO}_{3}$ ( $\mathrm{PMN}-\mathrm{PT}$ ), a prototypical ferroelectric relaxor. We show that the widely used concept of polar nanoregions as indiviudal static entities is incompatible with the available body of experimental diffuse scattering results.
We propose a simple phenomenological model that not only reproduce observed diffuse scattering in great details but also is coherent with the unusual dielectric properties ${ }^{4}$. Our model assumes a specific, slowly changing, displacement pattern of the lead ion, which is indirectly controlled by the low-energy acoustic phonons of the system. The model qualitatively explains the temperature, pressure, and electric field dependence of diffuse neutron- and x-ray scattering, as well as the existence of hierarchy in the relaxation times of these materials.

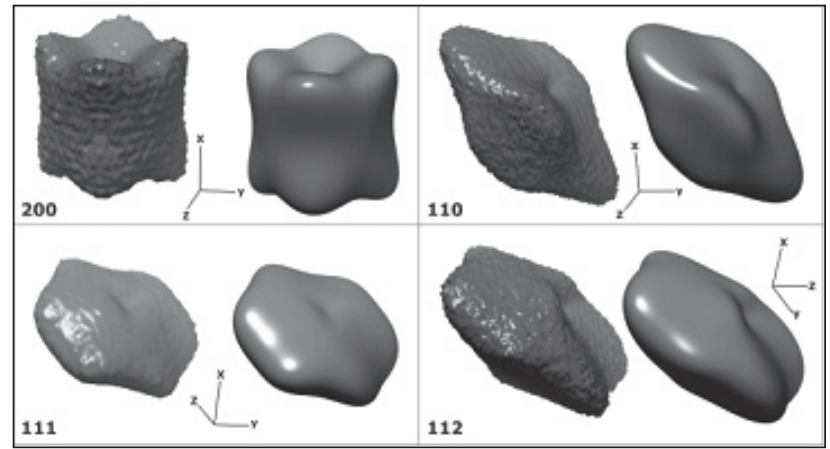

FIGURE. Comparison of experimental (PMN-PT) and model isosurfaces of diffuse scattering intensity in the proximity of various Bragg nodes.

[1] D. Viehland, S.J. Jang, L.E. Cross, M. Wutting, J. Appl. Phys. 1990, 68, 2916-2921. [2] G. Burns, B.A. Scott, Solid State Comm. 1973, 13, 423 -426 [3] G. Xu, G. Shirane, J.R.D. Copley, , P.M. Gehring, Phys. Rev. B 2004, 69, 064112-1 - 064112-6 [4] http://lanl.arxiv.org/abs/1101.0490

Keywords: relaxor ferroelectrics, diffuse scattering, perovskite structure

\section{MS.30.5}

Acta Cryst. (2011) A67, C78-C79

Diffuse scattering in one dimensional "liquid-like" aperiodic composites

Laurent Guérin, ${ }^{\mathrm{a}}$ Céline Mariette, ${ }^{\mathrm{a}}$ Bertrand Toudic, ${ }^{\mathrm{a}}$ Philippe Rabiller, ${ }^{a}$ Claude Ecolivet, ${ }^{\mathrm{a}}$ Mark Hollingsworth, ${ }^{\mathrm{b}}{ }^{a}$ Institut de Physique, Rennes, UMR 6251 CNRS Université de Rennes 135000 Rennes (France). 'Department of Chemistry, Kansas State University, Manhattan (USA). E-mail: laurent.guerin@univ-rennes1.fr

Uniaxial aperiodic composites are made of host opened nonstoechiometric structures confining guest molecules which posses their own periodicity [1], [2], [3]. From the crystallographic point of view, translation symmetry is recovered in higher dimensional superpaces, in agreement with the existence of non-periodic Bragg peaks, indexed with more than three indices [4]. This aperiodicity generates many kinds of disorder, which may concern self-correlation functions, pair correlation functions, molecular disorder [5]. They induce a very rich diffuse scattering pattern in the reciprocal space.

Here we will focus on the shortest alkane guest molecules within urea inclusion compounds. We have recently shown that with guest heptane to dodecane molecules, these materials present a high temperature phase with one dimensional order for the guest subsystem. Very original pattern of the associated diffuse planes are observed. At lower temperature, these crystals present unique phase ordering phase transitions, to higher dimensional crystallographic superspaces [6]. 